\title{
Expressed demand for healthcare services in regional South Australia: a cross-sectional study
}

\section{CURRENT STATUS: POSTED}

Research Square

Matthew Leach

University of South Australia

matthew.leach@unisa.edu.auCorresponding Author

ORCiD: https://orcid.org/0000-0003-3133-1913

\section{Sandra Walsh}

University of South Australia

Kuda Muyambi

University of South Australia

Marianne Gillam

University of South Australia

Martin Jones

University of South Australia

\section{DOI:}

$10.21203 / \mathrm{rs} .2 .13594 / \mathrm{v} 2$

\section{SUBJECT AREAS}

Health Economics \& Outcomes Research

\section{KEYWORDS}

expressed demand; health; health care; health needs assessment; needs; regional; rural; survey 


\section{Abstract}

The authors have withdrawn this preprint from Research Square. 